\title{
Las TIC y su uso en estudiantes universitarios. El caso de una universidad confesional
}

\author{
Francisco Sereño Ahumada*
}

\section{RESUMEN}

El presente artículo se enfocó en la investigación que tuvo como fin caracterizar a los estudiantes en el uso de las TIC de la UCSH, teniendo como base teórica las tecnologías de la información como causa y efecto de un sistema que sienta sus bases en la lógica de la globalización y la hiperconexión. A nivel metodológico, el estudio fue de naturaleza cuantitativa y la recolección de datos se efectuó mediante una encuesta. El análisis de resultados fue estadístico. En cuanto a los hallazgos, muestran que los estudiantes son más consumidores de tecnología que productor de conocimiento.

Palabras clave: Sociedad de la información, Tecnología de la Información y Comunicación, Informática educativa, Herramientas web, Estudiantes universitarios.

\section{ICT and its use in college students. The case of a denominational university.}

\section{ABSTRACT}

This article it approach on research that was aimed to characterize the students in the use of ICT, with the theoretical basis of the information technologies as cause and effect of a system that has its basis in the logic of globalization and the hyperconnection. A methodological level, the study was quantitative in nature and data collection was carried out through a survey. The statistical analysis of results was. As for the findings show that consumers are more students technology as a producer of knowledge.

Keywords: Information Society, Information Technology and Communication, Education Computing, Web tools, Undergraduates.

* Profesor, Magíster en Pedagogía Universitaria, Académico Dirección de Bachillerato y Propedéutico de la Universidad Católica Silva Henríquez, chileno, E-mail: fsereno@ucsh.cl 


\section{Introducción}

La inclusión de las Tecnologías de la Información y la Comunicación (TIC en adelante) ha generado cambios en las actividades de las personas y en el desarrollo económico de la sociedad. Mundialmente, no existe ámbito en que no esté participando una herramienta emanada de la informática o la electrónica, y la educación no ha sido la excepción, influenciada por las TIC y sus derivados en el ámbito pedagógico. En este caso, sus principales acciones han sido enfocadas hacia las dimensiones relacionadas con la calidad de los procesos de enseñanza, sus resultados en cuanto a aprendizaje (cuantitativa y cualitativamente) (Aguaded \& Cabero, 20I4), aspectos concernientes a pertinencia, actualización, ajustes de enfoques (sicológicos, curriculares, entre otros), etc.

De la misma forma, la utilización de estos recursos ha tenido un fuerte impacto en el trabajo académico, tanto al interior de las instituciones como fuera de ellas, que es el lugar donde se determinan muchas de las acciones que posteriormente se ejecutarán en las aulas. Esto en gran medida ha revalorizado el trabajo pedagógico, que con el apoyo de las TIC, ha hecho posible vislumbrar contextos potenciales de acción académica, que hace un tiempo atrás no eran posibles (Barroso Osuna \& Cabero Almenara, 2013).

Esta incorporación de las nuevas tecnologías a la actividad docente ha llevado a un trabajo cada vez más exigente, desde el punto de vista de lo que se debe realizar para mantener el nivel de calidad de las actividades y las expectativas que ello genera en los estudiantes, quienes viven la tecnologización con naturalidad respecto de los profesores.

Aun a pesar de la envergadura de las posibilidades y cambios generados por la red, el factor humano está presente, el desafío actual es encontrar la forma de organizar las ideas, los objetivos y los procesos para alcanzar aprendizajes en el contexto actual. En tal sentido, la Globalización ha permitido que disciplinas concebidas en esferas de pensamiento y acción, muy lejanas a la educación, surtan de herramientas distintas a las usadas históricamente en el área de la generación de competencias en estudiantes (Coll \& Monereo, 2008b). 
Consciente de esta situación, se planteó la siguiente pregunta de investigación: ¿Qué conocimientos en el manejo de las TIC tienen los estudiantes de la Universidad Católica Silva Henríquez y cuáles utilizan como recurso para el aprendizaje? Para responder a esta pregunta, se planteó el siguiente objetivo general: "Caracterizar el uso de las TIC como medio de aprendizaje en estudiantes de la UCSH”.

La metodología ha sido planteada desde el paradigma analítico explicativo, con un diseño descriptivo cuantitativo. La técnica de recolección de datos fue a través de una encuesta, aplicada a estudiantes universitarios de la UCSH, y el tratamiento de la información es estadístico no inferencial. Los datos se analizaron de forma descriptiva a través de tablas de frecuencias. Estos análisis tuvieron como propósito responder a las preguntas de investigación formuladas, con el fin de develar una caracterización en el uso de las TIC en los estudiantes UCSH.

\section{Antecedentes y marco teórico}

Las Tecnologías de la Información y Comunicación (TIC) han introducido elementos transformadores para el desarrollo de los estudiantes en su encuentro con el aprendizaje:

Las TIC permiten un nuevo escenario para la enseñanza y aprendizaje. Basados en las oportunidades que ofrecen éstas, los autores que apoyan este argumento abogan por cambios radicales en la manera en que los estudiantes aprenden y en la que los profesores enseñan, promoviendo prácticas de enseñanza constructivistas, centradas en los alumnos, con compromiso activo, interacción permanente, diálogo, etcétera. (Hinostroza \& Labbé, 20II, p. I0).

La evidencia acumulada señala que la sociedad ha contribuido en los últimos cincuenta años en transformaciones radicales a nivel sociocultural y sociopolítico (Castells, 1998; Coll \& Monereo, 2008a; UNESCO, 2005). Estas aplicaciones, que integran medios de informática, telecomunicaciones y redes, posibilitan tanto la comunicación y colaboración interpersonal (persona a persona) como la multidireccional (uno a muchos o muchos a muchos). "Estas 
herramientas desempeñan un papel sustantivo en la generación, intercambio, difusión, gestión y acceso al conocimiento" (Cobo, 2009, p. 312); y tal como lo plantea Libedinsky (2016), las tecnologías son una aliada para el aprendizaje y para la interacción social, puesto que son un medio para la indagación, la exploración, la expresión, la colaboración y la comunicación con el otro.

A nivel educativo, se han modificado los procesos cotidianos y esto se debe a la inclusión de sistemas de información, y como consecuencia, ha habido un proceso transformador en el ámbito de los procesos de aprendizaje, lo que se traduce en la integración de las nuevas tecnologías en todos los ámbitos del currículum, y por ende, en los proyectos educativos y planes estratégicos de cada institución de educación escolar y superior cambiando la forma de comunicación y transmisión de la información (Ernesto \& Arauz, 2015).

La instalación y masificación de estas nuevas formas de comunicación y obtención de información se han producido gracias a Internet, la velocidad y dinámica de expansión han permitido establecer progresivamente políticas tecnológicas para la educación en países tercermundistas y en vías de desarrollo; internet genera expectativas similares a la televisión en cuanto a transformar y potenciar la educación (Cobo \& Moravec, 20II; Urías, Torres, Valdés \& Serna, 20I5). En Latinoamérica se han implementado políticas de mediano y largo plazo y creado estándares tecnológicos, con el propósito de mejorar la calidad de la educación en lugares rurales y aislados de la urbe, para, de esta forma, superar la brecha digital que se instala como consecuencia negativa de nueva perspectiva digital.

Estos cambios de políticas responden a la obligación del Estado a considerar que las nuevas generaciones de jóvenes tienen una competencia innata que desarrolla formas de investigar otras fuentes de información a través de las TIC, por su cualidad de "nativos digitales" (Prensky, 2010) o bien como "Visitantes/Residentes" (White \& Le Cornu, 201I). Las antiguas generaciones se relacionan de manera distinta con las nuevas formas de comunicarse, deben aprehenderlas como un elemento ajeno a su formación. Los últimos son los llamados "inmigrantes informáticos”, ya que no tienen una vinculación directa con la tecnología y los procesos cognitivos que ésta supone. 
Lo anterior es evidencia de la consolidación de un cambio de perspectivas, por esto la sociedad actual en su conjunto ha identificado a la educación como un instrumento indispensable para lograr una participación equitativa y activa en el mundo globalizado, sin embargo, muchos estudiantes están lejos de ser parte de esa clase global de "nativos digitales" conectados a las redes sociales que utilizan las herramientas web para un aprendizaje efectivo, y -por otro lado-los docentes no pueden asumir que todo joven estudiante es un "nativo digital" que entiende y utiliza las TIC para apoyar y mejorar sus procesos de aprendizaje (Reig, 2013).

Siguiendo en esta línea, en Chile se implementa desde la década de los 90 un proceso de reforma educacional que incorpora el desarrollo digital a través de la red Enlaces I para cumplir con el propósito básico de que cada niño y adolescente reciba una educación de calidad y equidad, requisito para ser protagonista del desarrollo de la sociedad. Para esto, se incorporó transversalmente en el currículum el desarrollo tecnológico, cuyo objetivo es: "proveer a todos los alumnos y las alumnas de las herramientas que les permitirán manejar el mundo digital y desarrollarse en él en forma competente" (Mineduc, 2005, p. 27).

En este tenor, los estudiantes que nacieron en la era digital evidencian en su quehacer académico formas distintas de comunicación, interacción y técnicas de estudio, lo que hace que el mundo académico se enfrente al desafío de entender los alcances de aquello, en términos de cómo estas prácticas colaboran con la formación tradicional en el aula universitaria para el logro de aprendizajes y en el desarrollo de habilidades para la formación de estos profesionales, que -a su vez- trabajarán en un futuro con nuevas tecnologías de la información (Prensky, 20I I).

Ante esto, el desarrollo que han tenido las TIC ha complejizado los procesos cognitivos, obligando a redefinir el significado de muchos términos como: cercanía, multitarea, presencia, el contacto entre personas, etcétera. La mayor cantidad de información, de reflexiones y opiniones sobre la propia información, la inmediatez de los medios de comunicación interpersonales, la posibilidad de colaborar en proyectos mundiales sin moverse del entorno cercano y la capacidad

I http://www.enlaces.cl 
global de difusión de ideas y de conocimiento, hacen necesarias nuevas habilidades que permitan gestionar y valorar convenientemente la información para poder convertirla en conocimiento. Existe hoy, el contexto donde practicar con el alumno la gestión de los cambios y el aprendizaje para toda la vida (Delors, I996; Unesco, 2005).

\section{Metodología}

El trabajo desarrolló un estudio cuantitativo, estructurado, de corte transversal descriptivo-analítico, que tuvo como propósito caracterizar el uso de las TIC en estudiantes universitarios de las distintas carreras de la Universidad Católica Silva Henríquez. Este tipo de estudio permite distribuir la realidad, aportando un conocimiento de tipo censal o estadístico acerca de aquellos aspectos del objeto de estudio que resultan medibles desde una perspectiva numérica, buscando "especificar las propiedades, las características y los perfiles de personas, grupos, comunidades, procesos, objetos o cualquier otro fenómeno que se someta a un análisis" (Hernández Sampieri, Fernández Collado \& Baptista Lucio, 20I0, p. 28).

Para la recogida de datos se aplicó un instrumento constituido por una encuesta seccional descriptiva (Briones, 2012), a una población muestral integrada por 310 estudiantes de distintas carreras de la UCSH. La encuesta se diseñó con el fin de "fotografiar" en un momento dado el uso que los estudiantes de la UCSH dan a las TIC como recurso de aprendizaje, distribuyendo las variables en escala porcentual.

La investigación se centró en el contexto de que la sociedad actual experimenta profundos cambios a raíz del uso de las TIC en todos los ámbitos del quehacer humano. Cambios que afectan las costumbres de los individuos, hábitos, formas de trabajar y de comunicarse, entre otros, los cuales hacen que el estudiante modifique directamente su forma de aprendizaje.

La muestra para este estudio estuvo conformada de acuerdo al cálculo muestral, en donde el tamaño del universo será de 5600 estudiantes; con un $50 \%$ de heterogeneidad, un margen de error del $5 \%$ y un $95 \%$ de nivel de confianza, la que corresponde a 310 estudiantes, en cuanto a la cantidad de estudiantes que involucra las variables del presente estudio. 
Finalmente, la muestra estuvo compuesta por estudiantes universitarios de la UCSH, de la Región Metropolitana, con edades comprendidas entre 18 y 58 años. Las distribuciones de la muestra por género y carrera, se recogen en los figuras I y 2 , respectivamente.

Figura I: distribución general de la muestra de estudiantes por género

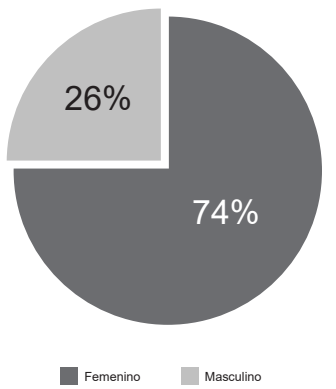

Fuente: Tasa de encuestados según su género a partir de datos recogidos

Figura 2: distribución general de la muestra de estudiantes por carrera

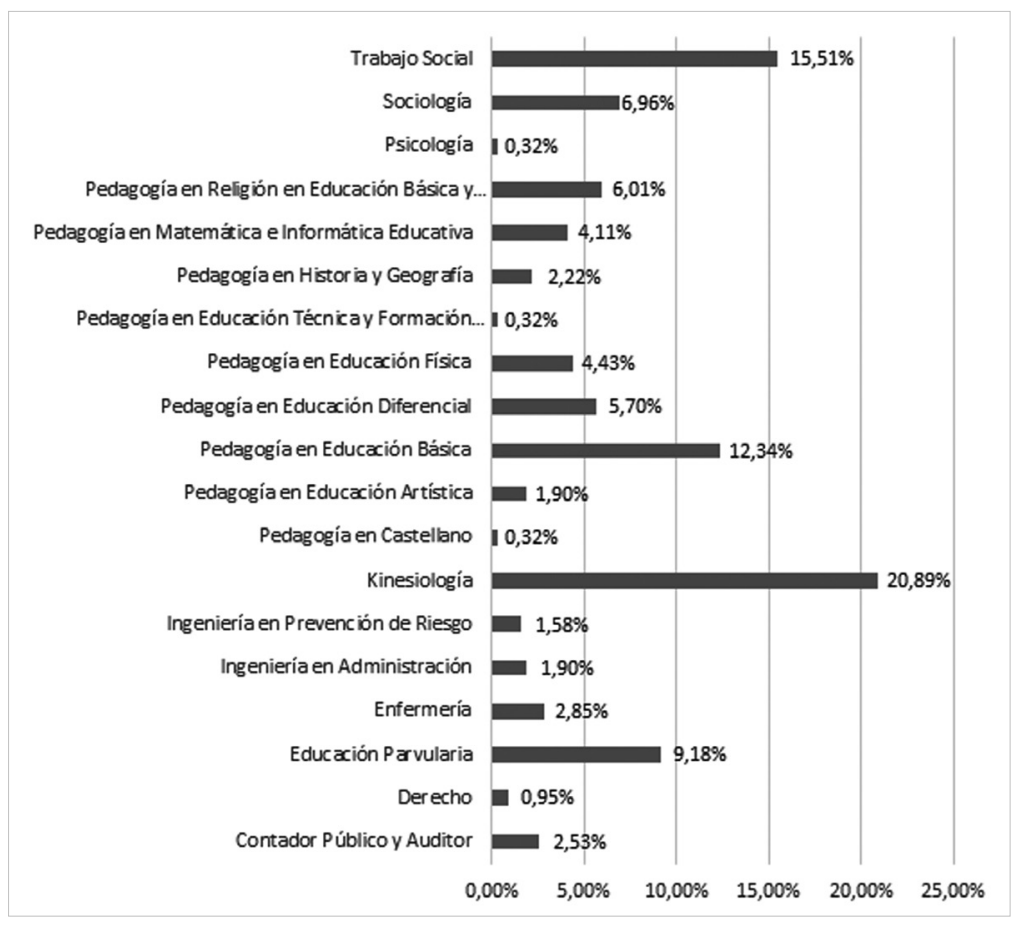

Fuente: Tasa de encuestados según distribución de carreras a partir de datos recogidos 
La descripción y distribución de la muestra permitió asegurar que la información es heterogénea, con sujetos de distintas carreras, años de formación y de docencia, lo que permitió asegurar la validez y confiabilidad de los datos recolectados por el instrumento.

\section{Resultados}

La presentación de resultados se realizó atendiendo solamente al análisis descriptivo. Para la obtención de los índices de los diferentes niveles de la escala de actitudes, se efectuó el cálculo de las puntuaciones de los ítems que integran cada nivel tomando en cada caso como puntuación de referencia el número de veces que los sujetos optaron por la respuesta codificada con un I "Siempre", 2 "A Veces", 3 "Nunca" y con un 4 "No se aplica", la cual se debe a la no respuesta al ítem. Se considera un 5I\% del valor total posible para cada nivel como punto de corte para identificar por arriba de éste "actitudes a favor" y por debajo "actitudes en contra".

Como las puntuaciones en una escala de Likert sólo tienen un significado relativo en función del conjunto de respuestas, es pertinente presentar los resultados estadísticos mediante tablas comparativas que permitan realizar estimaciones globales de contraste. Cabe señalar que la desviación estándar en algunos ítems no presenta mayor dispersión en las respuestas.

Tabla I: Tasas según respuestas de estudiantes

\begin{tabular}{|l|c|c|c|c|c|c|}
\hline \multicolumn{1}{|c|}{ USOS DE LAS TIC } & SIEMPRE & A VECES & NUNCA & $\begin{array}{c}\text { NO SE } \\
\text { APLICA }\end{array}$ & MEDIA & $\begin{array}{c}\text { DESVIACIÓN } \\
\text { ESTÁNDAR }\end{array}$ \\
\hline $\begin{array}{l}\text { Considero a Wikipedia } \\
\text { como una fuente válida de } \\
\text { información }\end{array}$ & $I 1,39 \%$ & $36,08 \%$ & $46,20 \%$ & $6,33 \%$ & 2,47 & 0,78 \\
\hline $\begin{array}{l}\text { Utilizo sistemas de chat para } \\
\text { comunicarse con mis amigos } \\
\text { o compañeros en Facebook, } \\
\text { Messenger o Whatsapp }\end{array}$ & $91, I 4 \%$ & $6,96 \%$ & $1,58 \%$ & $0,32 \%$ & $\mathrm{I}, \mathrm{II}$ & 0,39 \\
\hline $\begin{array}{l}\text { Utilizo portales de } \\
\text { bibliotecas virtuales tales } \\
\text { como: Biblioteca Miguel de } \\
\text { Cervantes, SIBUM, entre } \\
\text { otros, para realizar trabajos } \\
\text { académicos }\end{array}$ & $33,54 \%$ & $39,87 \%$ & $21,84 \%$ & $4,75 \%$ & $\mathrm{I}, 98$ & 0,86 \\
\hline
\end{tabular}




\begin{tabular}{|l|l|l|l|c|c|c|}
\hline \multicolumn{1}{|c|}{ USOS DE LAS TIC } & SIEMPRE & A VECES & NUNCA & $\begin{array}{c}\text { NO SE } \\
\text { APLICA }\end{array}$ & MEDIA & $\begin{array}{c}\text { DESVIACIÓN } \\
\text { ESTÁNDAR }\end{array}$ \\
\hline $\begin{array}{l}\text { Subo documentos para } \\
\text { trabajar en línea con mis } \\
\text { compañeros en Google- } \\
\text { Docs. }\end{array}$ & $38,61 \%$ & $29,75 \%$ & $26,58 \%$ & $5,06 \%$ & 1,98 & 0,93 \\
\hline $\begin{array}{l}\text { Utilizo herramientas de } \\
\text { sincronización on-line } \\
\text { como Windows Sync, } \\
\text { Dropbox, u otros }\end{array}$ & $38,29 \%$ & $34,49 \%$ & $22,15 \%$ & $5,06 \%$ & 1,94 & 0,90 \\
\hline $\begin{array}{l}\text { Me conecto a Internet para } \\
\text { buscar información en } \\
\text { Google Académico relativa } \\
\text { a mis estudios }\end{array}$ & $60,13 \%$ & $34,81 \%$ & $4,11 \%$ & $0,95 \%$ & 1,46 & 0,62 \\
\hline $\begin{array}{l}\text { Consulto videos en sitios } \\
\text { web tales como: Youtube, } \\
\text { Vimeo o Livestream } \\
\text { para responder a mis } \\
\text { inquietudes académicas }\end{array}$ & $62,34 \%$ & $29,43 \%$ & $6,96 \%$ & $1,27 \%$ & 1,47 & 0,68 \\
\hline $\begin{array}{l}\text { Busco información en los } \\
\text { foros o blogs para construir } \\
\text { mi aprendizaje }\end{array}$ & $25,32 \%$ & $43,99 \%$ & $25,32 \%$ & $5,38 \%$ & 2,11 & 0,84 \\
\hline $\begin{array}{l}\text { Utilizo la web para } \\
\text { descargar documentos que } \\
\text { favorecen mi aprendizaje }\end{array}$ & $76,58 \%$ & $19,94 \%$ & $3,16 \%$ & $0,32 \%$ & 1,27 & 0,53 \\
\hline $\begin{array}{l}\text { Subo archivos multimedia } \\
\text { para compartir con mis } \\
\text { compañeros }\end{array}$ & $41,46 \%$ & $36,39 \%$ & $16,77 \%$ & $5,38 \%$ & 1,86 & 0,88 \\
\hline
\end{tabular}

Fuente: distribución porcentual a partir de datos recogidos

Una vez establecida la tabla de resultados, se puede develar que la web es el recurso más utilizado a través de las redes sociales, esto tiene explicación puesto que los jóvenes utilizan dispositivos móviles en su conexión a través de wifi o red de datos, los que también son una plataforma de identidad digital (Barroso Osuna \& Cabero Almenara, 2013). Por otro lado, el uso de Wikipedia no es tan frecuente en los estudiantes de la UCSH, sin embargo, las redes sociales abarcan sobre el 90\% de preferencias. Ahora, si bien es cierto que existen portales como rincondelvago, monografías u otras, solo un 39,8\% declaran a veces utilizar portales de bibliotecas virtuales para la realización de trabajos académicos, seguido de un 33,5\% que sí ocupa estas bibliotecas y solo un $21,8 \%$ señala que no las utiliza para efectuar trabajos académicos. 
A estos datos se suman las herramientas provistas por Google que han permitido que muchos usuarios puedan hacer prescindible el uso de ciertos procesadores textos, pero si se relaciona con los datos obtenidos, un 4I,4\% de estudiantes declaran que "siempre" suben documentos para trabajarlos con sus compañeros en línea, seguido de un $36,3 \%$ que señala hacerlo de vez en cuando y solo un I6,7\% dice no hacerlo nunca, pero un 5,38\% declara no saber hacerlo.

\section{Discusión y conclusiones}

El uso de las TIC se relaciona actualmente, como indicaron Villa y Poblete (2007), con un aprendizaje basado en competencias y con la gestión de la información apoyada en las amplias tecnologías a las que dan acceso las aplicaciones y los distintos dispositivos tecnológicos. De manera genérica esto incluye: la gestión de archivos, edición de documentos, utilización del correo electrónico y las listas de distribución, navegación por internet, navegación y edición de blogs, consulta y aportaciones en wikis y en foros, elaboración de presentaciones, utilización de hojas de cálculo y participación en redes sociales temáticas o genéricas, entre otras acciones que incluyen el uso de los soportes informáticos.

Los hallazgos dan cuenta de que existe una percepción favorable por parte del estudiantado respecto a la utilidad que poseen las TIC para el apoyo a la formación profesional. En relación a esto, se observa un uso generalizado de dispositivos electrónicos como soporte para hacer actividades fuera del aula, así como la utilización masiva de Internet. Es interesante destacar que Google Académico es una herramienta conocida por los estudiantes universitarios y es usada por casi el $98 \%$ de los alumnos, arrojando un importante dato para quienes son docentes universitarios. Esta herramienta puede ser incorporada como parte de las exigencias de trabajos de investigación o fuente para presentaciones orales, ya que se trata de un recurso web que contiene muchos textos que no están en las bibliotecas y que, en algunos casos, son imposibles de adquirir por el alto precio que tienen.

En conjunto con esto, cabe destacar que es menor, aunque bastante generalizada, la utilización de Wikipedia y otros sitios como 
monografías.com como fuente válida de información. En este sentido, es pertinente decir que se observa una carencia en la formación investigativa entregada por los colegios de procedencia en la medida en que los alumnos no tienen totalmente incorporado como conducta de entrada el criterio de validación de fuente, cuestión relevante para encontrar información validada que sirva para generar conocimientos apropiados para la formación académica.

Debido a la potencia y versatilidad de estas tecnologías, su utilización puede servir como herramienta para el desarrollo y adquisición de otras competencias, como son la búsqueda, manejo y elaboración de información, reflexión y pensamiento crítico, habilidades comunicativas tanto escritas como gráficas, multimedia e incluso orales, capacidad de análisis y síntesis, planificación del tiempo, entre otras. Lo anterior se instala como un aporte a la transversalidad, ya que cuando se desarrolla una competencia transversal, se actúa en mayor o menor medida sobre muchas otras.

La educación se abre a nuevas maneras de ser conferida, a nuevas situaciones de enseñanza, que complementan la metodología clásica de la clase presencial y de la búsqueda bibliográfica en textos impresos. Según el programa de la OCDE Aprendiz del Nuevo Milenio:

Las implicancias de este fenómeno son infinitas. Es posible que el uso continuado de las tecnologías digitales tenga importantes implicaciones en el desarrollo de las competencias intelectuales y de las capacidades cognitivas, hasta el punto de que algunos analistas opinan que se termina por pensar de forma bien distinta (De Saint Pierre, 2009, p. 2).

Las principales aplicaciones se han enfocado en mejorar la calidad de los procesos de enseñanza, los resultados de aprendizaje (cuantitativa y cualitativamente) y aspectos concernientes a pertinencia, actualización, ajustes de paradigmas (sicológicos, curriculares, entre otros), etc. De la misma forma, la utilización de estos recursos como herramienta metodológica, ha tenido impacto en el trabajo del formador en el aula y fuera de esta, que es lugar donde se determinan muchas de las acciones académicas que posteriormente se ejecutarán. 
En el mismo punto se concluye que es pertinente incorporar como práctica académica emanada de los profesores, el conocimiento de portales como bibliotecas virtuales, a los cuales un tercio de los alumnos nunca han entrado. El dato que reafirma lo anterior, es el trabajo con las citas bibliográficas -fundamentales para la validez de los trabajos académicos-. Por otro lado, más de la mitad de los estudiantes no utiliza software para citas. ante lo cual se tensiona que ciertas metodologías docentes deberían fomentar el uso correcto de las mismas, en el entendido que esto se relaciona con la ética profesional y con aprender a partir de información verídica, además del fomento al respeto por el trabajo de otros.

Un recurso extendido, y que demuestra el nivel de responsabilidad respecto al manejo logístico y la gestión de la información, es la utilización de dispositivos de respaldo de la misma y la sistematización de la información en carpetas virtuales. El uso de estos sistemas que se pueden calificar de tradicionales, contrasta con el desconocimiento por parte de la mitad de los encuestados de formas de respaldo virtuales aparecidas en el mercado, como Dropbox, las cuales tienen la ventaja de no ser dispositivos físicos y tener mayor capacidad de almacenamiento.

Sin embargo, para que el estudiante use las tecnologías con fines educativos, será la institución educativa quien deberá formarlos como usuarios digitales activos, ya que así le darán un uso pedagógico a las TIC, permitiendo el levantamiento de redes comunicativas y de interacción con y entre personas de distintas partes del planeta, generando un potencial que favorecería la construcción del aprendizaje individual y social y el desarrollo de habilidades que permitan un aprendizaje autónomo. Además de ofrecer lo anteriormente descrito, las TIC abren posibilidades que apoyan los procesos de enseñanzaaprendizaje, generando motivación, interés, creatividad, imaginación y nuevos métodos de comunicación.

Las TIC tienen hoy una presencia permanente en el panorama educativo. Sin embargo, gran parte de sus usuarios potenciales desconocen o tienen una idea confusa acerca de sus posibilidades reales e implicancias educativas, quedándose solo con una parte de las utilidades que representa el uso de las TIC, es decir, solo le dan 
un uso personal y de ocio, que en muchos casos es solo de consumo de la información, sin darle un uso a los recursos. Entonces, se infiere que la labor de los docentes en este contexto es presentar, dirigir, discriminar, seleccionar, ordenar, orientar esos procesos comunicativos para transformarlos en procesos educativos. Según cómo el profesor realice estas actividades, es posible identificar enfoques metodológicos de enseñanza según la opción que cada docente tenga, por ejemplo, conductista, constructivista, etc. Es así como también el espectro de situaciones de enseñanza se ha diversificado (López Carrasco, 2013).

Los hallazgos dan cuenta de una caracterización en el uso de las TIC por parte de los estudiantes de la UCSH, los cuales plantean que el uso de las tecnologías es de carácter de consumidor más que de generador de aprendizaje, lo que plantea un desafío al docente, quien deberá plantear estrategias de aprendizaje en donde el uso de las TIC sea con el propósito de construir aprendizaje mediatizado por las tecnologías digitales.

Finalmente, y si bien es cierto que hay mucha investigación respecto a este tema, los resultados de la presente investigación dan los insumos para iniciar un estudio respecto a las formas en que los docentes incorporan las tecnologías de la información en el aprendizaje y construir un perfil de competencias digitales acordes a la realidad de la UCSH, entonces ¿cuáles y cómo son utilizados los recursos digitales en la sala de clase y qué impacto tienen en el aprendizaje?

\section{Referencias bibliográficas}

Aguaded, J. I. \& Cabero, J. (20I4). Tecnologías y medios para la educación en la e-sociedad. Madrid: Alianza Editorial.

Barroso Osuna, J. \& Cabero Almenara, J. (20I3). Nuevos escenarios digitales. Las tecnologías de la información y la comunicación aplicadas a la formación y desarrollo curricular (I ed.). Madrid: Ediciones Pirámide.

Briones, G. (2012). Métodos y técnicas de investigación para las ciencias sociales (4 ed. Vol. I). México: Trillas.

Castells, M. (I998). La Era de la Información. Economía, Sociedad y Cultura. (Vol. II: El Poder de la Identidad). Madrid: Alianza Editorial. 
Cobo, C. (2009). El concepto de tecnologías de la información. Benchmarking sobre definiciones de las TIC en la sociedad del conocimiento. Revista de estudios de comunicación - Zer, I4(27), 295-318.

Cobo, C. \& Moravec, J. (20II). Aprendizaje invisible. Hacia una nueva ecología de la educación. Barcelona: Publicacions i Edicions de la Universitat de Barcelona.

Coll, C. \& Monereo, C. (2008a). Educación y aprendizaje en el siglo XXI: Nuevas berramientas, nuevos escenarios, nuevas finalidades. En C. Coll \& C. Monereo (Eds.), Psicología de la educación virtual. Madrid: Ediciones Morata.

Coll, C. \& Monereo, C. (2008b). Psicología de la educación virtual: aprender y enseñar con las Tecnologías de la Información y la Comunicación. Madrid: Ediciones Morata.

De Saint Pierre, D. (2009). Aprendices del nuevo milenio, el desafío de la educación en el Siglo XXI. Recuperado de http://www.polisdigital.cl/content/ view/552647/Aprendices-del-nuevo-milenio-el-desafio-de-laeducacion-del-siglo-2I.html

Delors, J. (1996). La Educación encierra un tesoro. Madrid: Ediciones UNESCO.

Ernesto, F. \& Arauz, R. (20I5). TIC en Educación. Escenarios y experiencias. Distrito Federal de México: Ediciones Díaz de Santos.

Hernández Sampieri, R., Fernández Collado, C. \& Baptista Lucio, M. d. P. (2010). Metodología de la Investigación (5 ed.). Mexico Distrito Federal: McGraw Hill.

Hinostroza, J. E. \& Labbé, C. (20I I). Políticas y prácticas de informática educativa en América Latina y el Caribe. Serie Políticas Sociales (pp. 84). Santiago: Comisión Económica para América Latina y el Caribe.

Libedinsky, M. (2016). Cómo y para qué integrar la tecnología a la enseñanza universitaria. Recuperado de http://www.academia.edu/24805240/ C \% C $3 \%$ B 3 mo_y_para_qu\%C 3\% A ${ }_{-}$integrar_la_ tecnolog\%C3\%ADa_a_la_ense\%C3\%BIanza_universitaria

López Carrasco, M. Á. (2013). Aprendizaje, competencias y TIC: aprendizaje basado en competencias ( $\mathrm{I}$ ed.). México: Pearson Educación.

MINEDUC(2005). Curriculum de la Educación Media. Objetivos Fundamentalesy Contenidos Mínimos Obligatorios. Recuperado de http://www.agenciaeducacion.cl/ wp-content/uploads/2013/02/MarcoCurriculardeEducMedia.pdf

Prensky, M. (2010). Nativos e inmigrantes digitales. Recuperado de http://www. marcprensky.com/writing/Prensky-NATIVOS E INMIGRANTES DIGITALES (SEK).pdf

Prensky, M. (20I I). Enseñar a nativos digitales. Madrid: Ediciones SM. 
Reig, D. (2013). Los jóvenes en la era de la biperconectividad: Fundación Telefónica.

UNESCO (2005). Hacia las sociedades del conocimiento. París: Ediciones UNESCO.

Urías, M., Torres, M., Valdés, A. \& Serna, M. (2015). Teorías que sustentan la Tecnología Educativa. En J. Angulo, J. Vales, C. Acosta \& R. García (Eds.), Aportes y reflexiones sobre la educación mediada por tecnología (pp. 38 - 54). México Distrito Federal: Tabook Servicios Editoriales e Integrales.

Villa, A. \& Poblete, M. (2007). Aprendizaje Basado en Competencias. Una propuesta para la evaluación de las competencias genéricas. Bilbao: Ediciones Mensajero.

White, D. S. \& Le Cornu, A. (20II). Visitor and residents: A new typology for inline engagement. First Monday, I6(9). doi:http://dx.doi. org/I0.5210/fm.vi6i9.317I 